\title{
Structural changes induced by ligand binding drastically increase the thermostability of the Ser/Thr protein kinase TpkD from Thermus thermophilus HB8
}

Yusuke Fujino, Takero Miyagawa, Masayuki Torii, Masao Inoue, Yuki Fujii, Hiroki Okanishi, Yoshikatsu Kanai, Ryoji Masui

\begin{tabular}{|c|l|}
\hline \multicolumn{1}{|c|}{ Citation } & FEBS Letters. 595(2). 264-274. \\
\hline Issue Date & 2021-01-24 \\
\hline Version of Record & 2020-11-28 \\
\hline Type & Journal article \\
\hline Text version & Author \\
\hline Supporting & $\begin{array}{l}\text { Supporting Information is available at } \\
\text { https://doi.org/10.1002/1873-3468.13996. }\end{array}$ \\
\hline Rights & $\begin{array}{l}\text { This is the peer reviewed version of the following article: FEBS Letters. } \\
\text { Vol.595, Issu.2, 264-274., which has been published in final form at } \\
\text { https://doi.org/10.1002/1873-3468.13996. This article may be used for } \\
\text { non-commercial purposes in accordance with Wiley Terms and Conditions for } \\
\text { Use of Self-Archived Versions. This article may not be enhanced, enriched or } \\
\text { otherwise transformed into a derivative work, without express permission } \\
\text { from Wiley or by statutory rights under applicable legislation. Copyright } \\
\text { notices must not be removed, obscured or modified. The article must be linked } \\
\text { to Wiley's version of record on Wiley Online Library and any embedding, } \\
\text { framing or otherwise making available the article or pages thereof by third } \\
\text { parties from platforms, services and websites other than Wiley Online Library } \\
\text { must be prohibited. }\end{array}$ \\
\hline DOI & \begin{tabular}{l} 
10.1002/1873-3468.13996 \\
\hline
\end{tabular} \\
\hline
\end{tabular}

Self-Archiving by Author(s)

Placed on: Osaka City University Repository

Fujino, Y., Miyagawa, T., Torii, M., Inoue, M., Fujii, Y., Okanishi, H., Kanai, Y. and Masui, R. (2020) Structural changes induced by ligand-bidning drastically increase the thermostability of the Ser/Thr protein kinase TpkD from Thermus thermophilus HB8. FEBS Letter., 595(2), 264-274. 


\title{
Structural changes induced by ligand-binding drastically increase the thermostability of the Ser/Thr protein kinase TpkD from Thermus thermophilus HB8
}

Yusuke Fujino $^{1}$, Takero Miyagawa ${ }^{2}$, Masayuki Torii ${ }^{1}$, Masao Inoue ${ }^{3}$, Yuki Fujii ${ }^{1}$, Hiroki Okanishi $^{4}$, Yoshikatsu Kanai ${ }^{4,5}$ and Ryoji Masui ${ }^{1}$

${ }^{1}$ Graduate School of Science, Osaka City University, Osaka 558-8585, Japan

${ }^{2}$ Graduate School of Frontier Biosciences, Osaka University, Suita, Osaka 565-0871, Japan

${ }^{3}$ Graduate School of Agriculture, Kyoto University, Kyoto 606-8502, Japan

${ }^{4}$ Graduate School of Medicine, Osaka University, Suita, Osaka 565-0871, Japan

${ }^{5}$ Integrated Frontier Research for Medical Science Division, Institute for Open and

Transdisciplinary Research Initiative, Osaka University, Suita, Osaka 565-0871, Japan

\section{Correspondence}

Graduate School of Science, Osaka City University, 3-3-138 Sugimoto, Sumiyoshi-ku, Osaka 558-8585, Japan. Tel.: +81-6-6605-2819; E-mail: rmasui@sci.osaka-cu.ac.jp

\begin{abstract}
Thermophilic proteins maintain their structure at high temperatures through a combination of various factors. Here, we report the ligand-induced stabilization of a thermophilic Ser/Thr protein kinase. Thermus thermophilusTpkD unfolds completely at $55{ }^{\circ} \mathrm{C}$ despite the optimum growth temperature of $75^{\circ} \mathrm{C}$. Unexpectedly, we found that the TpkD structure is drastically stabilized by its natural ligands ATP and ADP, as evidenced by the increase in the melting temperature to $80^{\circ} \mathrm{C}$. Such a striking effect of a substrate on thermostability has not been reported for other protein kinases. Conformational changes upon ATP binding were observed in fluorescence quenching and limited proteolysis experiments. Urea denaturation of Trp mutants suggested that ATP binding affects not only the ATP-binding site, but also the remote regions. Our findings shed light on thermoadaptation of thermophilic proteins.
\end{abstract}




\section{Keywords:}

ATP binding, denaturation, protein kinase, substrate binding, thermophile, thermostability

\section{Abbreviations}

$\mathrm{CD}$, circular dichroism; $C_{\mathrm{m}}$, the midpoint concentration of chemical denaturation; PKAc, cAMP-dependent protein kinase catalytic subunit; STPK, Hanks-type serine/threonine protein kinase; $T_{\mathrm{m}}$, the midpoint temperature of thermal denaturation. 
The stability and dynamics of thermophilic and mesophilic proteins has been studied in detail over several decades. Some studies have reported that thermophilic proteins are more rigid than their mesophilic homologues [1,2], whereas others have shown the opposite $[3,4]$. This apparent discrepancy is derived from a variety of stabilizing forces, including greater hydrophobicity, more surface charges, increased hydrogen bonding and salt bridges, oligomerization, and complex formation with ligands [5-8].

Hanks-type Ser/Thr protein kinases (STPKs), also commonly named eukaryotic-like Ser/Thr protein kinases, have been well studied [9]. STPKs are model systems for studying the dynamic properties and allosteric regulation of proteins $[10,11]$. The core of STPKs is composed of two domains: the N-lobe dominated by a five-stranded $\beta$-sheet and the $\mathrm{C}$-lobe consisting of mostly $\alpha$-helices [11]. Two ensembles of hydrophobic residues, the C-spine and the R-spine, mediates the flexible connection between the lobes. Both spines are dynamically assembled: especially the C-spine is completed when the ATP binds to a deep cleft between the lobes. Through the activation process, STPKs achieve a specific configuration that allows the phosphotransfer to occur. Although correlation between conformational dynamics and activity in STPKs has been established, most of the previous researches have focused on mesophilic STPKs. In particular, stability and its relation to the dynamics of STPKs has not yet been examined well enough.

Thermophilic bacteria also have STPKs, which are structurally and functionally similar to eukaryotic STPKs $[12,13]$. Thermophilic proteins serve as model systems for understanding thermodynamic aspects of proteins. Therefore, the physicochemical characterization of thermophilic STPKs is expected to provide important insights into the relationship between the stability and dynamics of STPKs. However, no biophysical studies have been reported for thermophilic STPKs.

In this study, we focused on TTHA1370 (TpkD) of T. thermophilus HB8, an aerobic Gram-negative eubacterium that grows at temperatures ranging from 50 to $82^{\circ} \mathrm{C}$ [14]. This protein is one of four STPKs encoded in the T. thermophilus HB8 genome. We investigated phosphorylation of proteins in this bacterium $[15,16]$ and also characterized its protein kinases. During our biochemical studies, we discovered that the recombinant TpkD has unusually low thermostability as a T. thermophilus protein. We further found that ATP or ADP binding dramatically increased the thermostability of TpkD. We also examined the effect of ATP on urea denaturation of TpkD and some mutants. Our studies have implications for understanding the ligand-induced stabilization of protein in thermophiles. 


\section{Material and methods}

\section{Protein overexpression and purification}

The expression plasmid of TpkD (pET-HisTEV/ttha1370) was constructed by ligating the amplified ttha1370 fragment into the NdeI and BamHI sites of pET-15b-modified pETHisTEV vector [17] and introduced into Escherichia coli Rosetta2(DE3) (Novagen, Madison, WI, USA). The transformant was cultured at $37^{\circ} \mathrm{C}$ for $24 \mathrm{~h}$ in an overexpression medium as previously described [17]. After centrifugation, the harvested cells (10 g) were ultrasonicated in buffer I (20 mM Tris-HCl, pH 9.0, and $200 \mathrm{mM} \mathrm{NaCl}$ ). After centrifugation, proteins in the supernatant were loaded onto a TALON metal affinity column (TaKaRa, Shiga, Japan) and equilibrated with buffer I. After washing with buffer I, the bound proteins were eluted with $200 \mathrm{mM}$ imidazole in buffer I. The fractions containing the target protein were precipitated with $40 \%$ saturation ammonium sulfate. After centrifugation, the precipitate was dissolved with buffer I, the solution was treated with TEV protease at $25^{\circ} \mathrm{C}$ for $24 \mathrm{~h}$, and loaded onto a TALON column. The flow-through fractions were collected, precipitated with ammonium sulfate, and dissolved in buffer I. The solution was loaded onto a HiLoad 16/600 Superdex 75 pg column (GE Healthcare Biosciences, Piscataway, NJ, USA) on an ÄKTA explorer system (GE Healthcare Biosciences). The fractions containing the target protein were concentrated using a Vivaspin concentrator (Sartorius AG, Göttingen, Germany) and stored at $4^{\circ} \mathrm{C}$. The His-tag was removed by TEV protease as previously described [17]. The sample without the TEV protease treatment was designated as His6-TpkD. The concentrations of the purified proteins were determined by using molar absorption coefficients, calculated to be $29,841 \mathrm{M}^{-1} \mathrm{~cm}^{-1}$ at $278 \mathrm{~nm}$ for TpkD [18]. Autophosphorylation activity of the purified protein was confirmed by incorporating radiolabeled phosphate from $\left[\gamma_{-}{ }^{32} \mathrm{P}\right]$ ATP into His6-TpkD (further details are provided in the Supplementary Methods).

The expression plasmids for W55F, W191F, and G57L mutants were constructed by QuikChange Site-Directed Mutagenesis (Stratagene, La Jolla, CA, USA), using pETHisTEV/ttha1370 as a template and primers (Table S1). The expression plasmid for the W93F mutant was prepared by inverse PCR. All mutant proteins were prepared using the same method as for the wild-type protein.

\section{Circular dichroism (CD) spectroscopy}


CD measurements were carried out with a Jasco spectropolarimeter, model J-720W. CD spectra of $3 \mu \mathrm{M}$ TpkD were measured in a 1-mm cell in the far-UV region between 200 and $250 \mathrm{~nm}$. Measurements were performed after incubating it at $25^{\circ} \mathrm{C}$ in $50 \mathrm{mM}$ Tris- $\mathrm{HCl}, \mathrm{pH}$ 7.5 , and $150 \mathrm{mM} \mathrm{NaCl}$. We assessed the thermostability by measuring $\mathrm{CD}$ values at $222 \mathrm{~nm}$ at a $1{ }^{\circ} \mathrm{C} / \mathrm{min}$ rate. Measurements were also performed in the presence of $5 \mathrm{mM} \mathrm{MgCl}_{2}$ and 1 mM adenine nucleotide (ATP, AMP-PNP, ADP, or AMP).

\section{Fluorescence spectroscopy}

The fluorescence emission spectra were measured with a Hitachi spectrofluorometer, model F-4500. All measurements were taken with an excitation wavelength of $295 \mathrm{~nm}$ in a 5 $\times 5-\mathrm{mm}$ quartz cuvette at $25^{\circ} \mathrm{C}$. The reaction mixture contained $50 \mathrm{mM}$ Tris-HCl, $\mathrm{pH} 7.5$, $150 \mathrm{mM} \mathrm{NaCl}$, and either $3 \mu \mathrm{M}$ TpkD or mutant proteins. Measurements were also made in the presence of $5 \mathrm{mM} \mathrm{MgCl}_{2}$ and $1 \mathrm{mM}$ ATP. For the titration experiment with 0-12 $\mu \mathrm{M}$ ATP, the fluorescence intensities at $351 \mathrm{~nm}$ were plotted against the ATP concentration and the dissociation constant $\left(K_{\mathrm{d}}\right)$ of TpkD for ATP was determined by fitting Equation 1 to the observed change of fluorescence intensity $(\Delta \mathrm{F})$ using Igor Pro (WaveMetrics, OR, USA).

$$
\Delta \mathrm{F}=0.5 \cdot \Delta \mathrm{F}_{0} \cdot\left[\left([\mathrm{E}]_{0}+[\mathrm{L}]_{0}+K_{\mathrm{d}}\right)-\left\{\left([\mathrm{E}]_{0}+[\mathrm{L}]_{0}+K_{\mathrm{d}}\right)^{2}-4[\mathrm{E}]_{0}[\mathrm{~L}]_{0}\right\}^{0.5}\right]
$$

where $\Delta \mathrm{F}_{0}$ is the molar fluorescence intensity of the TpkD-ATP complex, [E] $]_{0}$ and $[\mathrm{L}]_{0}$ are total concentrations of TpkD and ATP, respectively [19].

In quenching experiments, the reaction mixtures also contained $0-0.5 \mathrm{M} \mathrm{CsCl}$ or acrylamide. Quenching data were analyzed by the Stern-Volmer equation [20].

$$
F_{0} / F=K_{\mathrm{Sv}}[\mathrm{Q}]+1
$$

where $F_{0}$ and $F$ are the fluorescence intensities before and after the addition of quencher (concentration [Q]), and $K_{\mathrm{SV}}$ is the effective quenching constant.

\section{Limited proteolysis}

The TpkD $(20 \mu \mathrm{M})$, with or without $1 \mathrm{mM}$ ATP and $5 \mathrm{mM} \mathrm{MgCl}_{2}$, was treated with trypsin in $50 \mathrm{mM}$ Tris- $\mathrm{HCl}, \mathrm{pH} 7.5$, and $150 \mathrm{mM} \mathrm{NaCl}$ at a protein to protease molar ratio of 5:1 at $37^{\circ} \mathrm{C}$ for various time points. The reaction was stopped by adding the sample buffer and the digests were separated by SDS-PAGE. The proteolytic fragments were identified by LC-MS/MS and database searching (further details are provided in the Supplementary Methods). 


\section{D structure modeling}

A model structure of TpkD was predicted by the Robetta server (http://robetta.bakerlab.org/). Model structures of ATP-bound TpkD were predicted by a docking program, AutoDock Vina [21]. The predicted structures were compared with the crystal structure cAMP-dependent protein kinase catalytic subunit (PKAc) complexed with ATP (PDB ID: 1ATP), and the structure with the least root-mean-square deviation of ATP was selected as the best model.

\section{Urea denaturation}

$\mathrm{CD}$ and fluorescence measurements were performed after incubation at $4^{\circ} \mathrm{C}$ for $2 \mathrm{~h}$ in 50 $\mathrm{mM}$ Tris- $\mathrm{HCl}$, $\mathrm{pH} 7.5,150 \mathrm{mM} \mathrm{NaCl}, 3 \mu \mathrm{M}$ TpkD, $5 \mathrm{mM} \mathrm{MgCl}_{2}$, and the indicated urea concentrations. Measurements were also performed in the presence of $1 \mathrm{mM}$ ATP. Other conditions were the same as described above.

To analyze the fluorescence spectra, the average wavelength was calculated after collecting all the emission spectra (in the range of $310-450 \mathrm{~nm}$ ) as follows:

Average wavelength $=\Sigma\left(\lambda_{\mathrm{i}} \times I_{\mathrm{i}}\right) / \Sigma I_{\mathrm{i}}$

where $\lambda_{\mathrm{i}}$ is the emission wavelength and $I_{\mathrm{i}}$ is the fluorescence intensity [22].

For urea-induced denaturation, the spectral data were analyzed using a two-state or threestate transition model. The two-state transition model recognizes the native $(\mathrm{N})$ and the unfolded $(\mathrm{U})$ conformations. The average wavelength (fluorescence) or molar ellipticity at $222 \mathrm{~nm}(\mathrm{CD})$ were plotted against the urea concentration and the thermodynamic parameters were determined by fitting Equation 4 to the observed spectroscopic signal $\left(Y_{0}\right)$ change using Igor Pro (WaveMetrics).

$$
Y_{0}=\frac{Y_{N}+Y_{U} \exp \left(\frac{m_{U-N}[D]-\Delta G_{U-N}^{0}}{R T}\right)}{1+\exp \left(\frac{m_{U-N}[D]-\Delta G_{U-N}^{0}}{R T}\right)}
$$

where $R$ is the gas constant, $T$ is the absolute temperature $(\mathrm{K}), Y_{\mathrm{N}}$ and $Y_{\mathrm{U}}$ are the signal intensity for native and unfolded states, respectively, [D] is the urea concentration, $\Delta G^{0}{ }_{\mathrm{U}-\mathrm{N}}$ is the change in Gibbs free energy upon the total unfolding of the native state, and $m_{\mathrm{U}-\mathrm{N}}$ is an empirical constant corresponding to the slope of a plot of $\Delta G$ against [D].

The three-state transition model recognizes the native (N), intermediate (I), and unfolded (U) conformations. The thermodynamic parameters were determined by fitting Equation 5 to 
the observed signal intensities.

$$
Y_{0}=\frac{Y_{N}+Y_{I} \exp \left(\frac{m_{I-N}[D]-\Delta G_{I-N}^{0}}{R T}\right)+Y_{U} \exp \left(\frac{m_{I-N}[D]-\Delta G_{I-N}^{0}}{R T}\right) \exp \left(\frac{m_{U-I}[D]-\Delta G_{U-I}^{0}}{R T}\right)}{1+Y_{I} \exp \left(\frac{m_{I-N}[D]-\Delta G_{I-N}^{0}}{R T}\right)+\exp \left(\frac{m_{I-N}[D]-\Delta G_{I-N}^{0}}{R T}\right) \exp \left(\frac{m_{U-I}[D]-\Delta G_{U-I}^{0}}{R T}\right)}
$$

where $Y_{\mathrm{I}}$ is the signal intensity for intermediate state, $m_{\mathrm{I}-\mathrm{N}}$, and $m_{\mathrm{U}-\mathrm{I}}$ are m-values for the respective transitions.

\section{Results}

\section{Thermostability of TpkD is drastically increased by ATP}

TpkD (TTHA1370) is a 253-residue protein, which consists only of the catalytic core of STPK (Fig. 1). Most of the functionally important motifs were conserved in TpkD, except for Gly in the DFG motif, which was substituted with Asp (Fig. 1A). We further generated a model structure of TpkD by homology modeling using a Mycobacterium STPK (PknA; PDB ID, $4 \times 3 \mathrm{f}$ ) as a template (Fig. 1B). Sequence identity and the root mean square deviation of $\mathrm{C} \alpha$ atoms between them were $30 \%$ and $2.6 \AA$ (205 atoms), respectively. The predicted TpkD structure was composed of the N-lobe and C-lobe, which was similar to that of the core of eukaryotic STPKs including PKAc (cAMP-dependent protein kinase catalytic subunit). The $\mathrm{N}$-lobe and C-lobe were assembled by hydrophobic residues, which constitute C-spine and Rspine. It should be mentioned that Gly57, a non-hydrophobic residue, was situated between Val68 and Phe144 in the predicted R-spine. Autophosphorylation activity (Fig. S1) and weak ATPase activity (data not shown) were observed for His6-TpkD, indicating the purified TpkD was active. It should be noted that autophosphorylation was observed for His6-TpkD, but not for $\mathrm{TpkD}$, suggesting that $\mathrm{TpkD}$ phosphorylated amino acid residue(s) in the His-tag sequence, but not in the activation segment.

We used CD (circular dichroism) analysis to investigate thermostability of TpkD. The far-UV CD spectrum at $25^{\circ} \mathrm{C}$ had two negative peaks at around $210 \mathrm{~nm}$ and $225 \mathrm{~nm}$ (Fig. $2 \mathrm{~A}$ ), indicating the presence of $\alpha$-helix. The signals at $222 \mathrm{~nm}$, indicative of changes in helical content, diminished abruptly when the temperature was approximately $>55^{\circ} \mathrm{C}$ (Fig. 2B). The melting temperature $\left(T_{\mathrm{m}}\right)$ of $\mathrm{TpkD}$ was $50^{\circ} \mathrm{C}$. In the cooling process following the heating, the magnitude of the CD signals was not restored (data not shown), indicating that 
the thermally induced unfolding was irreversible. This result was unexpected because the optimum temperature for growth of T. thermophilus $\mathrm{HB} 8$ was 65 to $72^{\circ} \mathrm{C}$ [14].

Then we examined the effect of ligand on the thermostability of TpkD (Fig. 2B). Interestingly, in the presence of $1 \mathrm{mM} \mathrm{ATP}$ and $5 \mathrm{mM} \mathrm{MgCl}_{2}$, the $T_{\mathrm{m}}$ was drastically increased to $80^{\circ} \mathrm{C}$. The $T_{\mathrm{m}}$ values were 53 and $50^{\circ} \mathrm{C}$ in the presence of ATP alone and $\mathrm{MgCl}_{2}$ alone, respectively. In a fixed concentration of $5 \mathrm{mM} \mathrm{MgCl}_{2}$, the increase in $T_{\mathrm{m}}$ was dependent on the ATP concentration and saturated at $50 \mu \mathrm{M}$ ATP (Fig. 2C).

Furthermore, we examined the effect of other adenine nucleotides on the thermostability of TpkD (Fig. 2D). In the presence of $1 \mathrm{mM}$ ADP, the $T_{\mathrm{m}}$ also increased to $84^{\circ} \mathrm{C}$, showing a similar effect to ATP. AMP-PNP, an unhydrolyzable ATP analogue, showed a significant but smaller stabilizing effect $\left(T_{\mathrm{m}}=67^{\circ} \mathrm{C}\right)$. In contrast, in the presence of $1 \mathrm{mM}$ AMP, the $T_{\mathrm{m}}$ was $52^{\circ} \mathrm{C}$, showing no significant stabilizing effect.

We did not monitor thermal unfolding process of TpkD by fluorescence spectroscopy since fluorescence intensity decreases strongly with temperature.

\section{ATP binding induces structural change in TpkD}

To reveal how ATP stabilizes TpkD and results in an increase in the $T_{\mathrm{m}}$, we used several different methods to analyze structural changes of TpkD. In the intrinsic (Trp) fluorescence emission spectra of TpkD excited at $295 \mathrm{~nm}$, the intensity of the peak (351 nm) increased in the presence of ATP and $\mathrm{MgCl}_{2}$ (Fig. 3A). No shift was observed in the maximum wavelength. The increase in the peak intensity was dependent on the ATP concentration (Fig. 3B). The data could be fitted to a hyperbolic equation describing a bimolecular binding reaction, suggesting that the ATP binding caused the spectral change. The dissociation constant $\left(K_{\mathrm{d}}\right)$ of ATP for TpkD was determined to be $0.5 \mu \mathrm{M}$. This value was similar to the $K_{\mathrm{m}}$ of ATPase by other protein kinases [23]. Since the fluorescence properties of Trp residues are sensitive to the microenvironment of fluorophores in proteins, this could suggest that local and/or global changes occurred in the tertiary structure of TpkD upon ATP binding. In contrast, the far-UV CD spectra showed no significant difference from those without nucleotides, suggesting no change in the secondary structure (Fig. 2A).

Quenching experiments with $\mathrm{CsCl}$ and acrylamide also suggested a conformational change of TpkD upon ATP binding. The intrinsic fluorescence intensity of TpkD decreased when the quenchers were added both in the absence and presence of ATP (Figs. 3C and D). For $\mathrm{CsCl}$, the slope of the Stern-Volmer plot was smaller in the presence of ATP than in its 
absence (Fig. 3C). In contrast, no significant difference in the plots for acrylamide were observed between in the absence and presence of ATP (Fig. 3D). The slope of the SternVolmer plot reflects largely the quenching of the more accessible residues. These suggested that ATP binding decreased the accessibility of a Trp residue probably through conformational change.

Furthermore, limited proteolysis supported conformational changes of TpkD upon ATP binding. The presence of ATP altered the proteolysis pattern of TpkD with trypsin, compared with that in its absence (Fig. 3E). The fragments were observed as relatively discrete bands on the gel in the presence of ATP, but not in its absence. Mass spectrometric analysis revealed that the about $20 \mathrm{kDa}$ fragment contained the region of residues 26-212 (Fig. S2), which encompass most of the $\mathrm{N}$ - and C-lobes. These results suggested that ATP binding caused certain conformational changes that increased resistance of TpkD to trypsin digestion.

\section{ATP also increases stability against urea-mediated denaturation of TpkD}

ATP also had a stabilizing effect on the chemical denaturation of TpkD as well as the thermal denaturation. We first studied the effect on TpkD of increasing the urea concentration by fluorescence measurements (Fig. 4). When the urea concentration was increased, the intrinsic fluorescence intensity of TpkD gradually decreased both in the absence and presence of ATP (Fig. 4A and B). However, the presence of ATP increased the midpoint concentration $\left(C_{\mathrm{m}}\right)$ values of urea in the denaturation curves based on the fluorescence intensities at $350 \mathrm{~nm}$ (Fig. 4C). The average wavelengths plotted against urea concentrations showed the effect of ATP more clearly (Fig. 4D). In the absence of ATP, the urea-induced denaturation of $\mathrm{TpkD}$ was considered as a three-state transition: the average wavelength was blue-shifted in 4-6 M urea, and then red-shifted above 6.0 M urea. However, when ATP was added to TpkD, only the single transition from 6.5-8.0 M urea was observed, indicating a two-state transition. In other words, the first transition at around $4.5 \mathrm{M}$ urea without ATP was almost diminished in its presence. These results suggested that conformational changes by ATP binding stabilized TpkD against urea denaturation. Since dilution experiments indicated the urea-induced unfolding was reversible, the thermodynamic parameters of unfolding were determined by curve-fitting the wavelength shift data to a twostate or three-state transition model (Table S2).

A similar effect of ATP was observed in the CD profiles for urea-mediated denaturation (Fig. S3). In the absence of ATP, TpkD showed a three-state transition: the first $C_{\mathrm{m}}$ was $4.1 \mathrm{M}$ urea and the second $C_{\mathrm{m}}$ was $6.4 \mathrm{M}$ urea. When ATP was added to TpkD, the profile was 
changed to a two-state transition with $C_{\mathrm{m}}$ of $6.0 \mathrm{M}$ urea. Together with the fluorescence data, these results suggested that ATP affected the secondary and tertiary structural changes induced by relatively low urea concentrations.

\section{Effects of ATP binding reach the region including Trp191}

We generated mutants of these three residues, W55F, W93F, and W191F, to examine their respective contributions to the fluorescence changes in urea-mediated denaturation. Figure 5 shows the fluorescence spectral changes dependent on urea concentration in the absence or presence of ATP. All three mutants had lower fluorescence intensities than the wild type and in the absence of ATP showed a three-state transition in the profiles of the fluorescence intensity as well as the wild type (Figs. 5A, B, E, F, I, and J). The stabilizing effect of ATP was also observed for all three mutants (Figs. 5C, G, and K). However, W191F showed two obvious differences in the denaturation processes. In the absence of ATP, its stability was significantly lower than the wild type and the other two mutants, and in the presence of ATP it showed a three-state transition (Fig. 5K), in contrast to the others (Figs. 4C, 5C, and G). These results suggested that Trp191 and/or the surrounding region are important for structural stability and ATP-induced stabilization of TpkD.

The profiles of average wavelength provided evidence that the effect of ATP reached the Trp191-surrounding region. W55F and W93F showed a three-state transition in the absence of ATP, but a two-state transition in its presence (Figs. 5D and H). These behaviors were similar to those of the wild type. In contrast, W191F showed a two-state transition not only in the presence of ATP, but also in its absence (Fig. 5L). In other words, the first transition at around the $4 \mathrm{M}$ urea in the wild type profile (Fig. 4D) disappeared in the W191F profile without ATP. This indicated that Trp191 mainly contributed to the fluorescence change as the first transition. This further suggested that ATP binding caused some structural change in the region including Trp191. A set of thermodynamic parameters was obtained for these mutants based on the average wavelength data (Table S2).

\section{Discussion}

This is the first report on the biochemical characterization of a protein kinase from a thermophilic bacterium. Thermus thermophilus is an extreme thermophile, which grows optimally at $65-72^{\circ} \mathrm{C}[14]$. However, $\mathrm{CD}$ analysis indicated that $\mathrm{TpkD}$ was unfolded completely at $55^{\circ} \mathrm{C}$. Unexpectedly, we found that ATP and $\mathrm{Mg}^{2+}$ stabilized the TpkD structure: the $T_{\mathrm{m}}$ was increased to $80^{\circ} \mathrm{C}$. ADP, but not AMP, stabilized TpkD similarly, and 
AMP-PNP was less effective than ATP and ADP. These results imply that ATP and ADP, natural ligands for TpkD, can have stabilizing effects. More than $50 \mu \mathrm{M}$ ATP with $\mathrm{Mg}^{2+}$ was enough to stabilize TpkD above $70^{\circ} \mathrm{C}$. The $K_{\mathrm{d}}$ value of TpkD for ATP was determined to be approximately $0.5 \mu \mathrm{M}$, which is in a similar range to the $K_{\mathrm{m}}$ values of other protein kinases [23]. The intracellular ATP level in bacteria is reported to be $1-5 \mathrm{mM}$ [24], and the physiological ATP/ADP concentration ratio is $\sim 10-1000[25,26]$. Therefore, it is probable that TpkD exists in an ATP- or ADP-bound state in T. thermophilus cells and can retain its tertiary structure, and subsequently its activity, at its growth temperature.

Conformational changes in TpkD by ATP binding were suggested by the results of fluorescence quenching, limited proteolysis, and urea denaturation of Trp mutants. During the urea denaturation process of the wild type, the average fluorescence emission wavelength was blue-shifted and then red-shifted. However, in the presence of ATP, the blue shift was lost. Interestingly, the W191F mutant also lost this peak shift. These results suggest that ATP binding changes the microenvironments around Trp191, leading to the loss of the blue shift of the fluorescence. A TpkD model structure predicts that Trp191 is located in the C-lobe and far from the ATP-binding site. This suggests that ATP binding affects the stability of not only the ATP-binding site, but also the remote regions.

Such far-reaching influence might be ascribed to intrinsic dynamics of STPKs. In the core architecture of STPK, the C- and R-spines are anchored to the long hydrophobic "Fhelix" in the C-lobe [11]. In a model structure of TpkD, Trp191 is located in the helix corresponding to the "F-helix" (Fig. 1B). ATP binding completes the C-spine, which contains Ile188 and Met192 in the F-helix. Therefore, it is probable that ATP binding affects the conformation and stability around Trp191.

The observed drastic stabilization of TpkD by ATP might be partly due to the structural architecture intrinsic to STPKs. However, such a striking effect of a substrate on thermostability has not been reported previously for other STPKs. The $T_{\mathrm{m}}$ values of apoPKAc, $46.9^{\circ} \mathrm{C}$, is increased to $52.5^{\circ} \mathrm{C}$ with $\mathrm{Mg}$-ATP and to $54.2^{\circ} \mathrm{C}$ with an inhibitor $\mathrm{H} 89$ [27]. The $T_{\mathrm{m}}$ values of MEK $1,54.6^{\circ} \mathrm{C}$, is increased by $3.2^{\circ} \mathrm{C}$ with $\mathrm{Mg}$-AMPPNP and by $2.9^{\circ} \mathrm{C}$ with Mg-ADP [28]. Non-substrate ligand-induced stabilization has also been known for several STPKs. The $T_{\mathrm{m}}$ value of PDK1 was increased by $20^{\circ} \mathrm{C}$ with an inhibitor UCN-01 [29]. In the crystal structure of UCN-01-bound PDK1, this compound binds in the ATPbinding site and interacts with the hydrophobic residues in N- and C-lobes [30]. As UCN-01 is larger and more hydrophobic than ATP, the mechanism of its effect on PDK1 structure is likely to be different from that of ATP on TpkD. Therefore, the stabilization by ATP might be 
a specific mechanism for substantial stabilization of thermophilic STPKs. This stabilization mechanism also might be unique in the sense that a substrate induces stabilization. It should be noted that the putative gap (Gly57) in the R-spine was not associated with the drastic stabilization by ATP since the G57L mutant, in which Leu was expected to fill the gap, also showed the increased $T_{\mathrm{m}}$ value in the presence of ATP (data not shown).

Ligand-induced stabilization has been reported for other proteins [31]. Binding of 8anilinonaphthalene-1-sulfonic acid increases $T_{\mathrm{m}}$ of BSA from 59 to $80^{\circ} \mathrm{C}$ [32]. Binding of biotin to streptavidin increased the $T_{\mathrm{m}}$ from 75 to $112^{\circ} \mathrm{C}$ [33]. In the former case, binding the ligand to multiple sites is considered a major cause of the large stabilizing effect [34]. In the latter case, stabilization of the tetrameric form of streptavidin is thought to increase the $T_{\mathrm{m}}$ [33]. Therefore, it is possible to suppose that ligand binding be adopted as a stabilization mechanism for other proteins in thermophiles including T. thermophilus.

\section{Author contributions}

RM conceived and supervised the study; YF (Fujino), TM, MT, MI, YF (Fujii) and RM designed the experiments; YF (Fujino), TM, MT, and HO performed the experiments; HO and YK provided MS tools and reagents; YF (Fujino), TM, MT, HO, and RM analyzed the data; YF (Fujino), MI, YF (Fujii), HO, YK and RM wrote the manuscript.

\section{References}

1 Wolf-Watz M, Thai V, Henzler-Wildman K, Hadjipavlou G, Eisenmesser EZ and Kern D. (2004) Linkage between dynamics and catalysis in a thermophilic-mesophilic enzyme pair. Nat Struct Mol Biol 11, 945-949.

2 Závodszky P, Kardos J, Svingor Á and Petsko GA. (1998) Adjustment of conformational flexibility is a key event in the thermal adaptation of proteins. Proc Natl Acad Sci U S A 95, 7406-7411.

3 Fitter J and Heberle J. (2000) Structural equilibrium fluctuations in mesophilic and thermophilic a-amylase. Biophys J 79, 1629-1636.

4 Tehei M, Madern D, Franzetti B and Zaccai G. (2005) Neutron scattering reveals the dynamic basis of protein adaptation to extreme temperature. J Biol Chem 280, 4097440979.

$5 \quad$ Brandts JF and Lin LN. (1990) Study of strong to ultratight protein interactions using differential scanning calorimetry. Biochemistry 29, 6927-6940.

6 Kumar S, Tsai C-J and Nussinov R. (2000) Factors enhancing protein thermostability. Protein Eng 13, 179-191.

$7 \quad$ Pace CN and McGrath T. (1980) Substrate stabilization of lysozyme to thermal and 
guanidine hydrochloride denaturation. $J$ Biol Chem 255, 3862-3865.

Razvi A and Scholtz JM. (2006) Lessons in stability from thermophilic proteins.

Protein Sci 15, 1569-1578.

9 Kornev AP and Taylor SS. (2015) Dynamics-driven allostery in protein kinases.

Trends Biochem Sci 40, 628-647.

10 Huse M and Kuriyan J. (2002) The conformational plasticity of protein kinases. Cell 109, 275-282.

11 Taylor SS and Kornev AP. (2011) Protein kinases: evolution of dynamic regulatory proteins. Trends Biochem Sci 36, 65-77.

12 Janczarek M, Vinardell J-M, Lipa P and Karaś M. (2018) Hanks-type serine/threonine protein kinases and phosphatases in bacteria: roles in signaling and adaptation to various environments. Int J Mol Sci 19, 2872.

13 Stancik IA, Sestak MS, Ji B, Axelson-Fisk M, Franjevic D, Jers C, Domazet-Loso T and Mijakovic I. (2018) Serine/threonine protein kinases from bacteria, archaea and eukarya share a common evolutionary origin deeply rooted in the tree of life. $J \mathrm{Mol}$ Biol 430, 27-32.

14 Oshima T and Imahori K. (1974) Description of Thermus thermophilus (Yoshida and Oshima) comb. nov., a nonsporulating thermophilic bacterium from a Japanese thermal spa. Int J Syst Bacteriol 24, 102-112.

15 Takahata Y, Inoue M, Kim K, Iio Y, Miyamoto M, Masui R, Ishihama Y and Kuramitsu S. (2012) Close proximity of phosphorylation sites to ligand in the phosphoproteome of the extreme thermophile Thermus thermophilus HB8. Proteomics 12, 1414-1430.

16 Masui R, Takahata Y, Inoue M, Iio Y, Okanishi H, Kim K, Nakagawa N, Yura K and Kuramitsu S. (2014) Structural insights of post-translational modification sites in the proteome of Thermus thermophilus. J Struct Funct Genomics 15, 137-151.

17 Inoue M, Fukui K, Fujii Y, Nakagawa N, Yano T, Kuramitsu S and Masui R. (2017) The Lon protease-like domain in the bacterial RecA paralog RadA is required for DNA binding and repair. $J$ Biol Chem 292, 9801-9814.

18 Kuramitsu S, Hiromi K, Hayashi H, Morino Y and Kagamiyama H. (1990) Pre-steady state kinetics of Escherichia coli aspartate aminotransferase catalized reactions and thermodynamic aspects of its substrate specificity. Biochemistry 29, 5469-5476.

19 Cheng K and Koland JG. (1996) Nucleotide binding by the epidermal growth factor receptor protein-tyrosine kinase. Trinitrophenyl-ATP as a spectroscopic probe. J Biol Chem 271, 311-318.

20 Lakowicz JR. (2006) Principles of Fluorescence Spectroscopy, Plenum Press. New York.

21 Trott O and Olson AJ. (2010) AutoDock Vina: improving the speed and accuracy of docking with a new scoring function, efficient optimization and multithreading. $J$ Comput Chem 31, 455-461.

22 Gardner NW, Monroe LK, Kihara D and Park C. (2016) Energetic coupling between ligand binding and dimerization in Escherichia coli phosphoglycerate mutase.

Biochemistry 55, 1711-1723.

23 Knight ZA and Shokat KM. (2005) Features of selective kinase inhibitors. Chem Biol 12, 621-637.

24 Klein AH, Shulla A, Reimann SA, Keating DH and Wolfe AJ. (2007) The intracellular concentration of acetyl phosphate in Escherichia coli is sufficient for direct phosphorylation of two-component response regulators. J Bacteriol 189, 55745581.

25 Buckstein MH, He J and Rubin H. (2008) Characterization of nucleotide pools as a 
function of physiological state in Escherzchia coli. J Bacteriol 190, 718-726.

26 Meyrat A and von Ballmoos C. (2019) ATP synthesis at physiological nucleotide concentrations. Sci Rep 9, 3070.

27 Steichen JM, Iyer GH, Li S, Saldanha SA, Deal MS, Woods VL, Jr. and Taylor SS. (2010) Global consequences of activation loop phosphorylation on protein kinase A. $J$ Biol Chem 285, 3825-3832.

28 Smith CK and Windsor WT. (2007) Thermodynamics of nucleotide and non-ATPcompetitive inhibitor binding to MEK1 by circular dichroism and isothermal titration calorimetry. Biochemistry 46, 1358-1367.

29 Busschots K, Lopez-Garcia LA, Lammi C, Stroba A, Zeuzem S, Piiper A, Alzari PM, Neimanis S, Arencibia JM, Engel M et al. (2012) Substrate-selective inhibition of protein kinase PDK1 by small compounds that bind to the PIF-pocket allosteric docking site. Chem Biol 19, 1152-1163.

30 Komander D, Kular GS, Bain J, Elliott M, Alessi DR and Van Aalten DMF. (2003) Structural basis for UCN-01 (7-hydroxystaurosporine) specificity and PDK1 (3phosphoinositide-dependent protein kinase-1) inhibition. Biochem J 375, 252-262.

31 Waldron TT and Murphy KP. (2003) Stabilization of proteins by ligand binding: application to drug screening and determination of unfolding energetics. Biochemistry 42, 5058-5064.

32 Celej MS, Montich GG and Fidelio GD. (2003) Protein stability induced by ligand binding correlates with changes in protein flexibility. Protein Sci 12, 1496-1506.

33 González M, Argaraña C and Fidelio G. (1999) Extremely high thermal stability of streptavidin and avidin upon biotin binding. Biomol Eng 16, 67-72.

34 Togashi DM and Ryder AG. (2008) A fluorescence analysis of ANS bound to bovine serum albumin: binding properties revisited by using energy transfer. J Fluoresc 18, 519-526. 


\section{Figures legends}

Fig. 1. (A) Sequence alignment of TpkD, PknA of Mycobacterium tuberculosis, and human PKAc. The amino acid residues composed of C-spine and R-spine are colored yellow and red, respectively. "P site" indicates an autophosphorylation site. Trp residues are colored magenta. (B) A model structure of TpkD-ATP complex shown in two orthogonal views. The structure is shown in a schematic representation. The amino acid residues composed of C-spine and $\mathrm{R}$ spine are represented as a space-filling model. ATP is represented as a stick model.

Fig. 2. Thermostability of TpkD. (A) Far-UV CD spectra with or without $1 \mathrm{mM}$ ATP and 5 $\mathrm{mM} \mathrm{MgCl}$. (B) Heat denaturation curves (relative values of $[\theta]_{222}$ ) with or without $1 \mathrm{mM}$ ATP and/or $5 \mathrm{mM} \mathrm{MgCl}_{2}$. (C) Dependence of $T_{\mathrm{m}}$ values on ATP concentration with $5 \mathrm{mM}$ $\mathrm{MgCl}_{2}$. (D) Effects of various adenine nucleotides $(1 \mathrm{mM})$ on TpkD denaturation with $5 \mathrm{mM}$ $\mathrm{MgCl}_{2}$.

Fig. 3. Changes of TpkD structure upon ATP binding. (A) Intrinsic fluorescence spectra with or without $1 \mathrm{mM} \mathrm{ATP}$ and $5 \mathrm{mM} \mathrm{MgCl}_{2}$. (B) ATP-binding curve (relative fluorescence intensity at $351 \mathrm{~nm}$ ) based on titration measurements. (C, D) Stern-Volmer plots for CsCl (C) or acrylamide (D) quenching of TpkD fluorescence with or without $1 \mathrm{mM}$ ATP and 5 $\mathrm{mM} \mathrm{MgCl}$. (E) Limited proteolysis with trypsin in the absence (upper panel) or presence (lower panel) of $1 \mathrm{mM} \mathrm{ATP}$ and $5 \mathrm{mM} \mathrm{MgCl}_{2}$. The digests were separated by SDS-PAGE and stained with Coomassie brilliant blue.

Fig. 4. Fluorescence spectral changes in urea-induced denaturation of TpkD. (A, B) Emission spectra in the absence (A) and presence (B) of $1 \mathrm{mM}$ ATP. (C, D) Denaturation curves based on relative fluorescence intensity at $350 \mathrm{~nm}(\mathrm{C})$ and average wavelength (D) with or without $1 \mathrm{mM}$ ATP.

Fig. 5. Fluorescence spectral changes of TpkD mutants. (A, E, I) Emission spectra of W55F (A), W93F (E), and W191F (I) in the absence of ATP. (B, F, J) Emission spectra of W55F (B), W93F (F), and W191F (J) in the presence of ATP. (C, G, K) Denaturation curves based on relative fluorescence intensity at $350 \mathrm{~nm}$ of W55F (C), W93F (G), and W191F (K). (D, H, L) Denaturation curves based on average wavelength of W55F (D), W93F (H), and W191F (L). 


\section{A}

Tt TpkD 1 MSLVGKTLSGRYRVVRPLARGALARVYLAFDP-FGTPYALKLFPPKARP - . - . RRDRE 53 $\begin{array}{lrl}M \text { PRA } & 2 & \text { SPRVGVTLSGRYRLQRLIATGGMGQVWEAVDNRLGRRVAVKVLKSEFSSDPEF IERFRAE } \\ \text { Hs PKAC } & 32 & \text { TPSQNTAQLDQFDRIKTLGTGSFGRVMLVKHKESGNHYAMKILDKQKVVKLKQIEHTLNE }\end{array}$

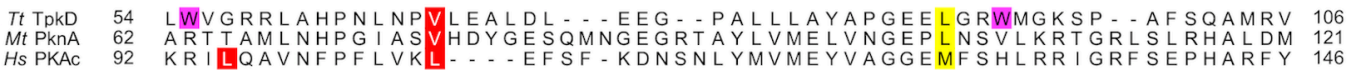
Tt TpKD 107 FHQ Activation segment 17 107 F HQLLLALAHMHEKGLVHRDVKPEN I LVNA-GEARLLDFDLSGPA- - QERFQKPLLLGTP 163 Hs PKAC 147 AAQIVLTFEYLHSLDL IYRDLKPENLL I DQQGY IQVTLFGFAKRVKGR . - . TWTLCGTP 202 HRD motif $\quad$ DFG motif

Tt TpkD 164 AYLAPELLRGLPSGPEADVYAAG I I LYWMLTGEHPFADPSGQVS - LDPDRGPHPPVA-GL 221 Mt PKחA 182 QY A A EQALGHDASPASDVYSLGVVGYEAVSGKRPFAGDGALTVAMKHIKEPPPPLPPDL 241

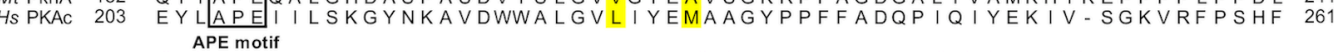

Tt TpkD 222 GEEAALYLERLLAPDPKARFPTAQEALKAFPF 253 HS PKAC 262 SSDLKDLLRNLLQVNPATRRF

C-spine R-spine
B

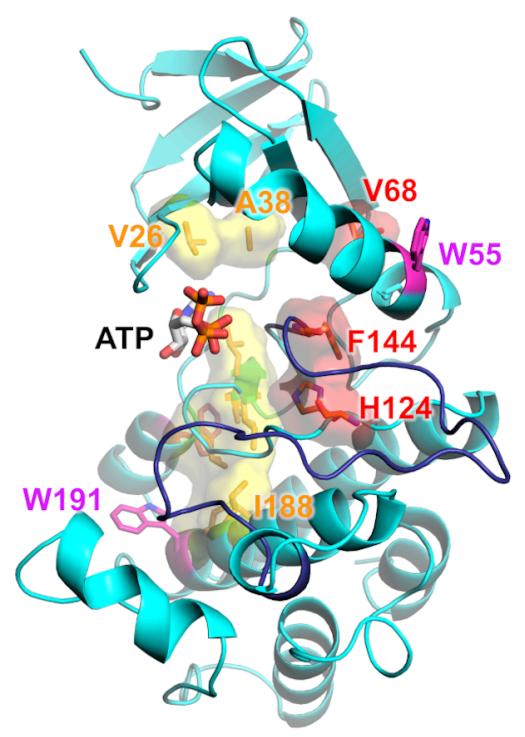

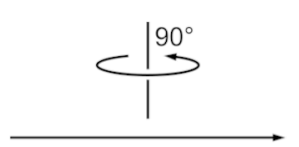

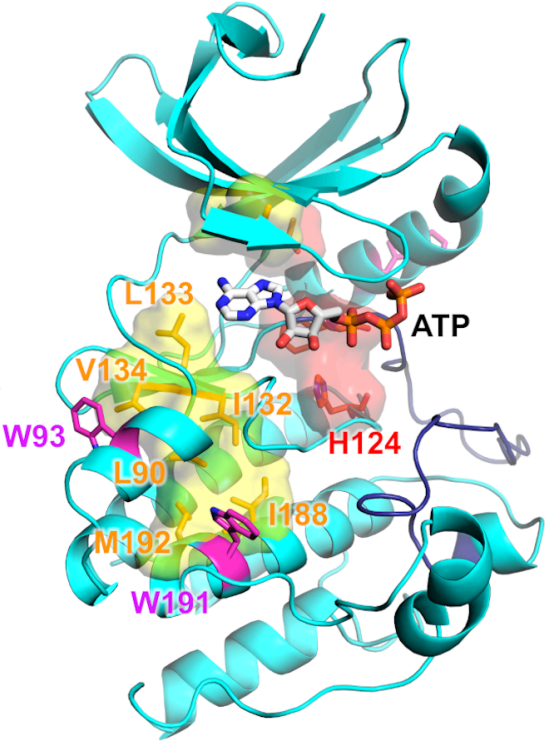

Figure 1 
A

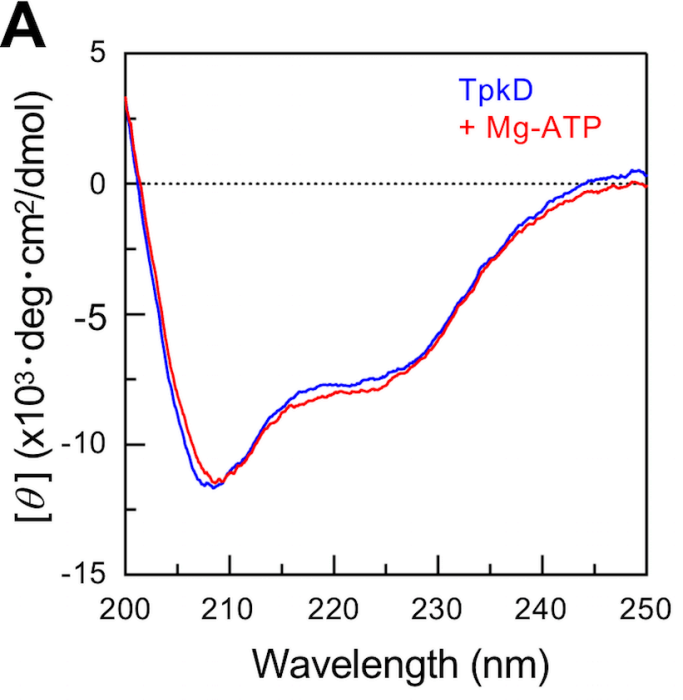

C

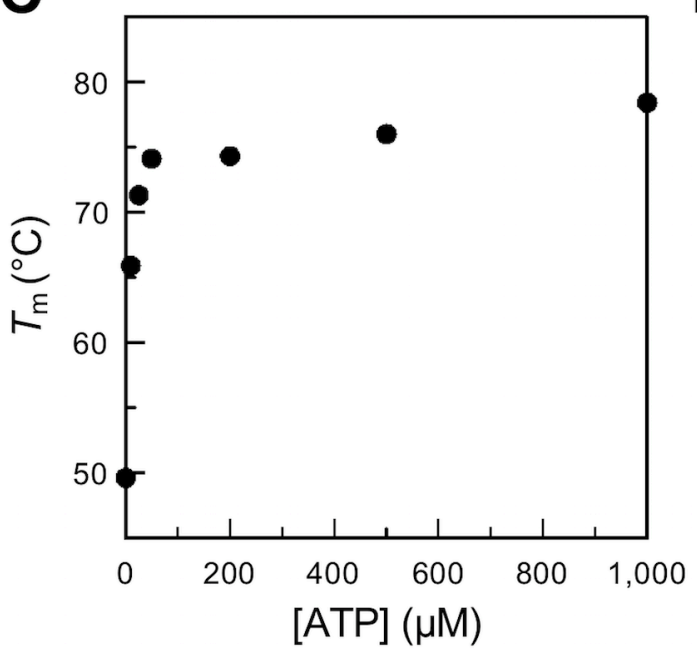

B

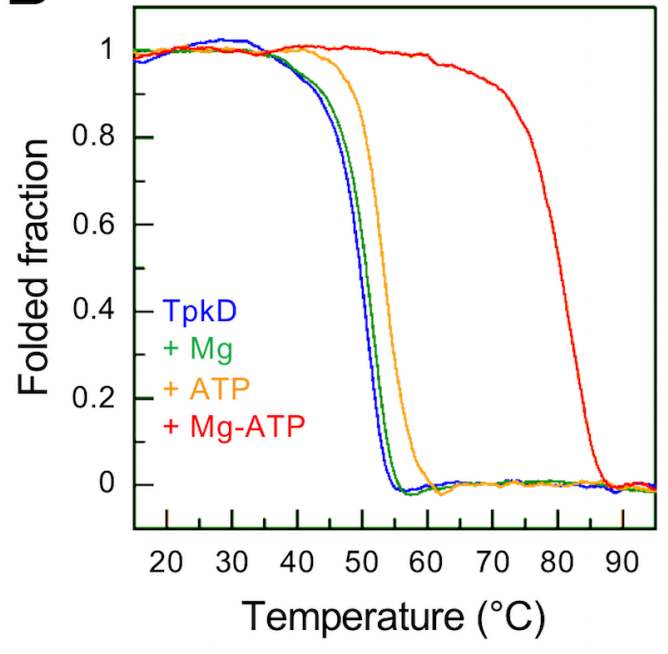

D

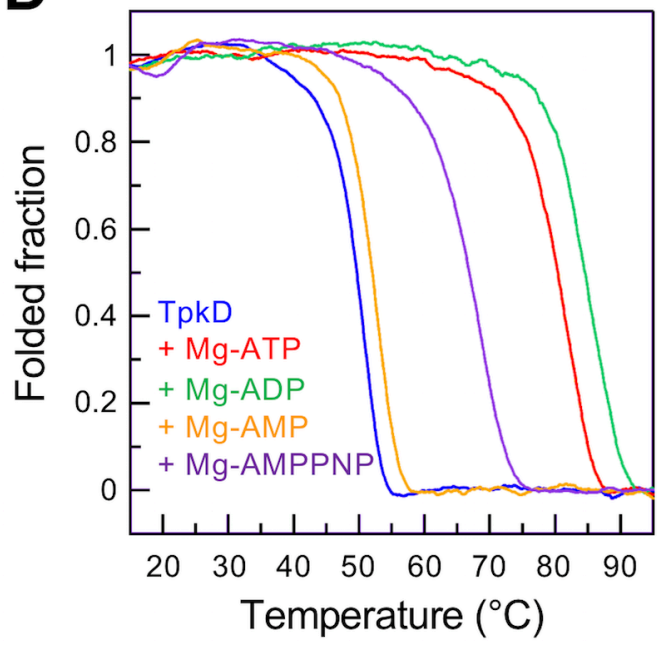

Figure 2 

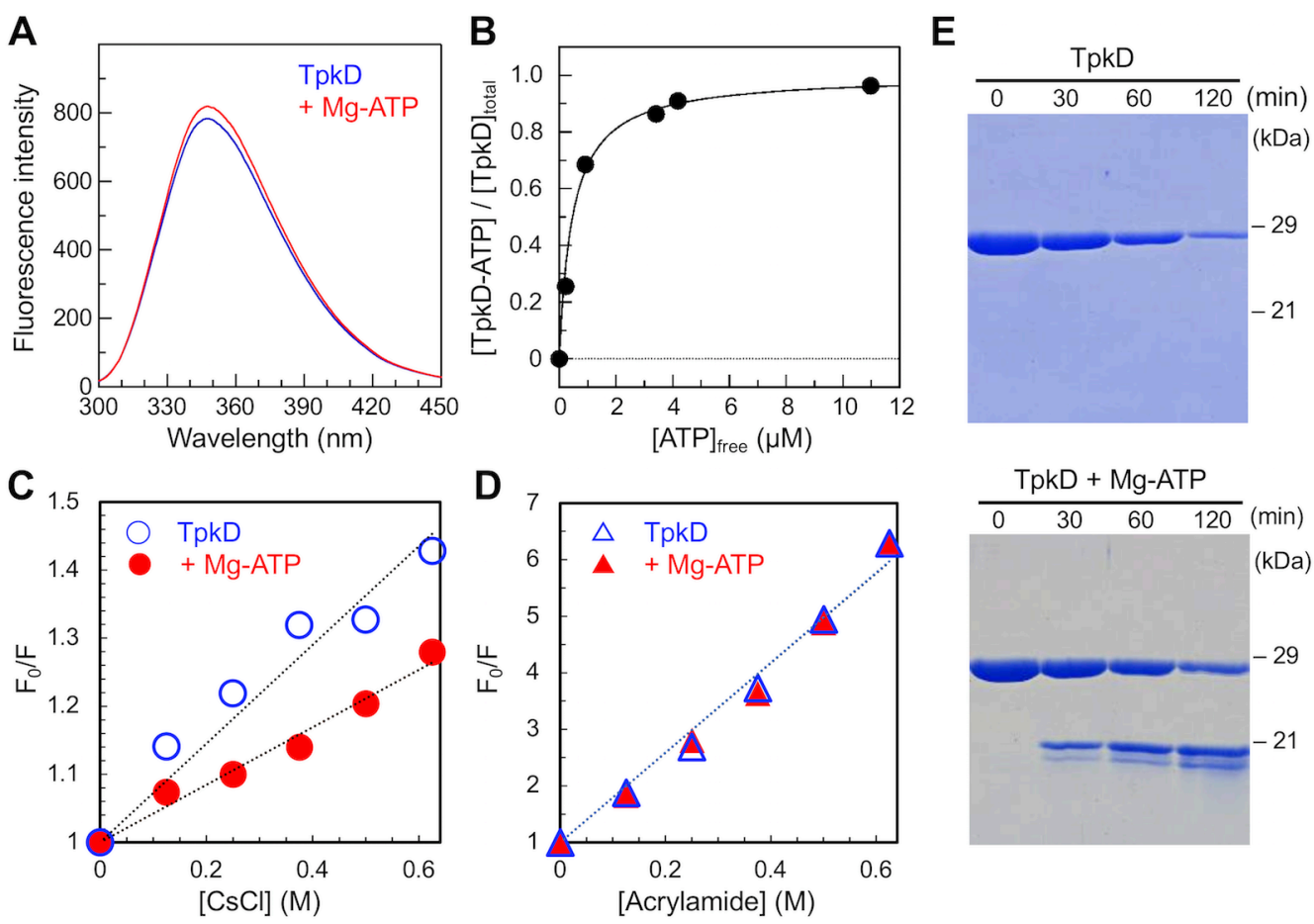

Figure 3 
A

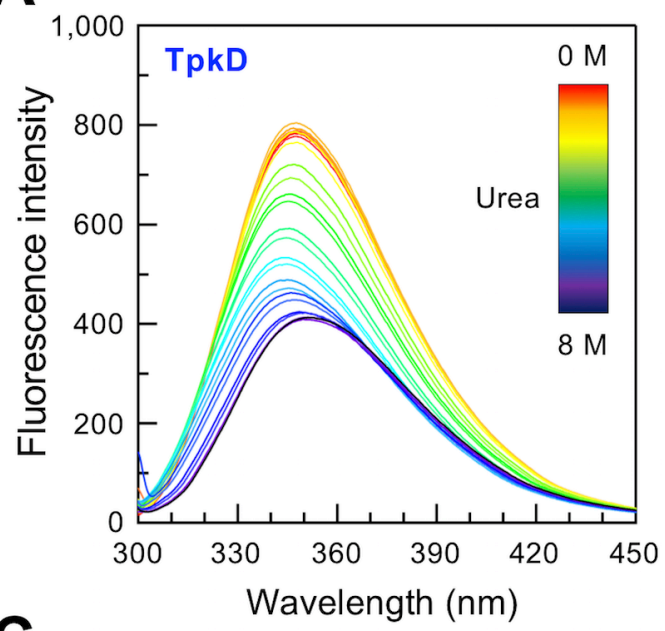

C

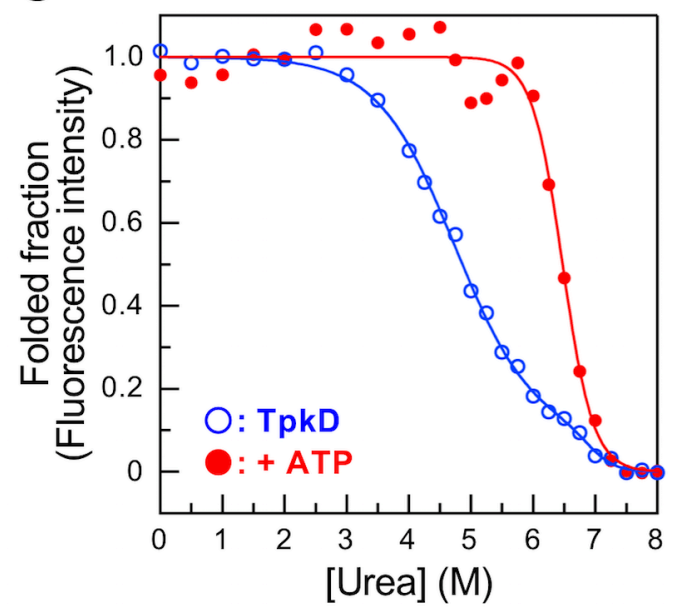

B

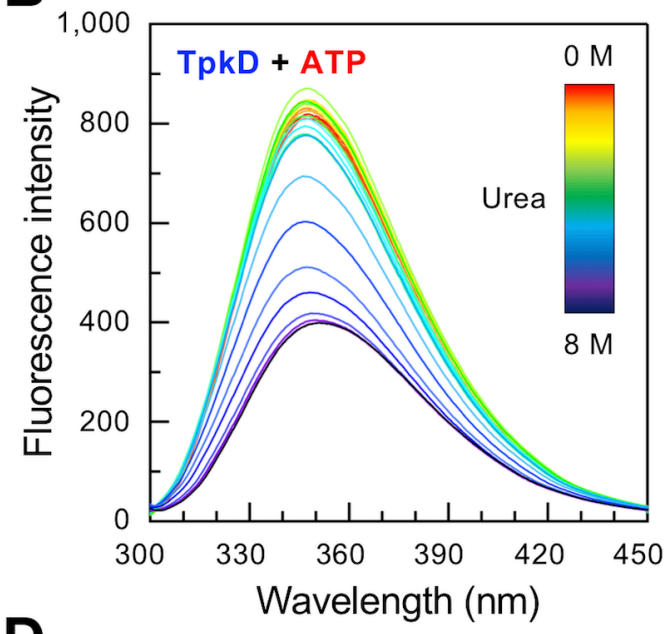

D

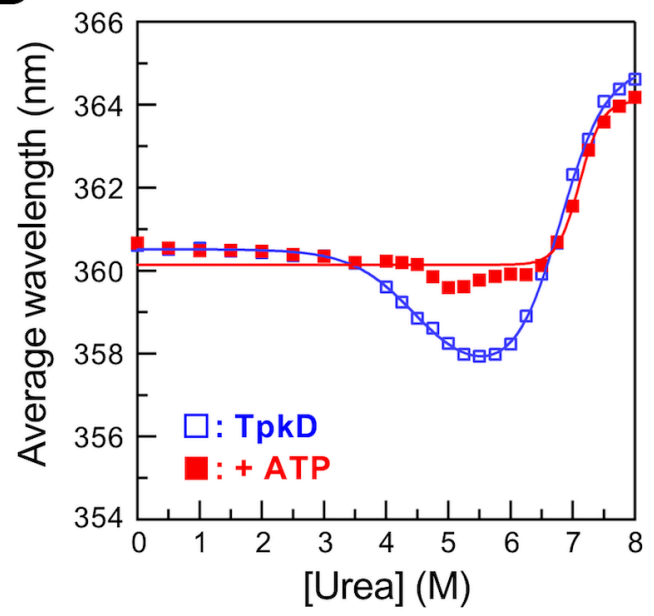

Figure 4 

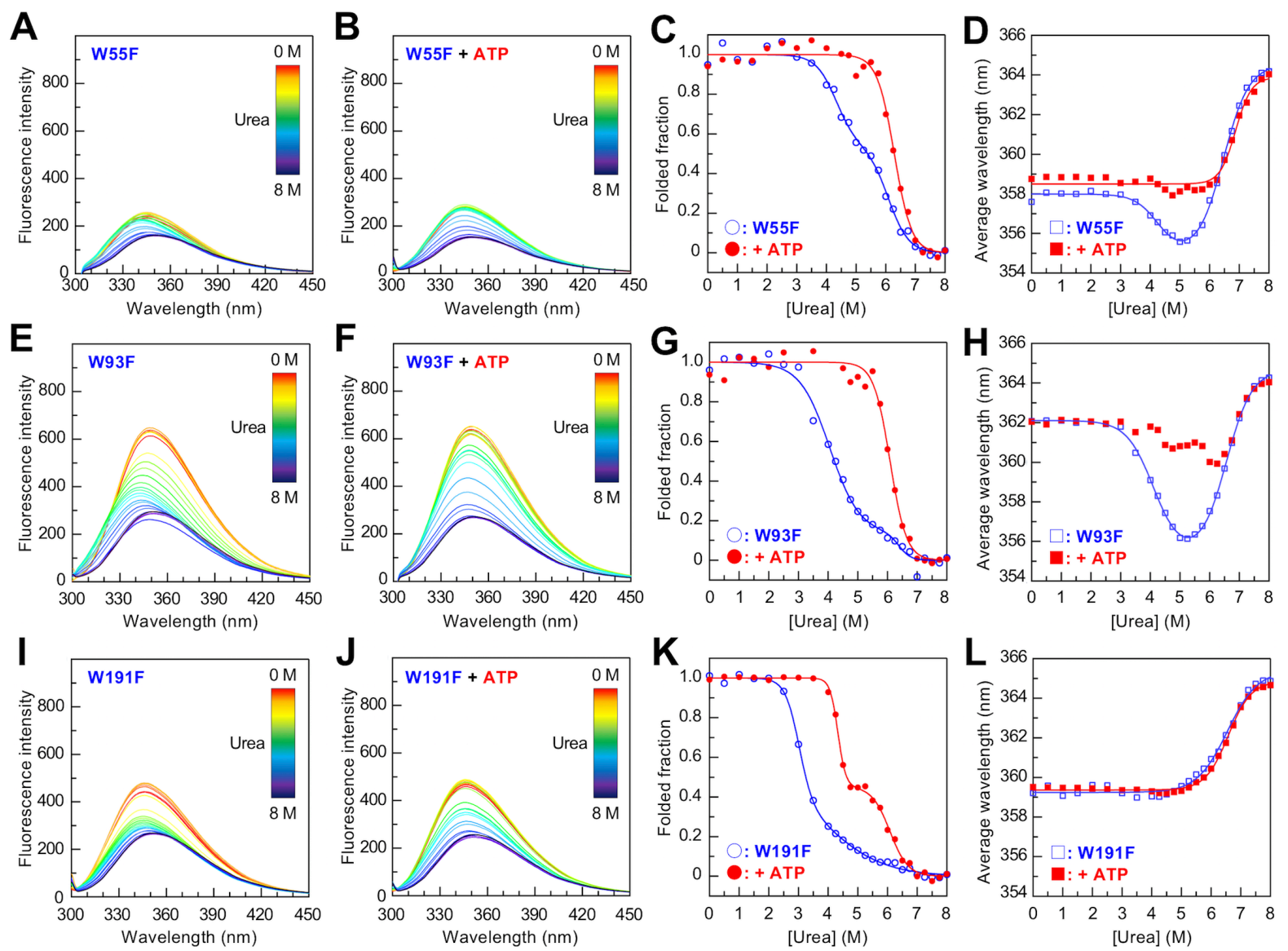

Figure 5 


\section{Structural changes induced by ligand binding drastically increase the thermostability of the Ser/Thr protein kinase TpkD from Thermus thermophilus HB8}

Yusuke Fujino, Takero Miyagawa, Masayuki Torii, Masao Inoue, Yuki Fujii, Hiroki Okanishi, Yoshikatsu Kanai and Ryoji Masui

\section{Supplementary Methods}

\section{Autophosphorylation assay}

For measurement of autophosphorylation activity, the reaction mixture contained $50 \mathrm{mM}$ Tris-HCl, $\mathrm{pH} 7.5,150 \mathrm{mM} \mathrm{NaCl}, 5 \mathrm{mM} \mathrm{MgCl} 2,3 \mu \mathrm{M}$ TpkD or His6-TpkD, and $10 \mu \mathrm{M}$ ATP containing $0.1 \mu \mathrm{C}_{\mathrm{i}} / \mu \mathrm{L}\left[\gamma^{32}\right.$-P]ATP. Reactions were performed at $37^{\circ} \mathrm{C}$ for $21 \mathrm{~h}$. After adding the sample buffer for SDS-PAGE and heat-treatment at $95^{\circ} \mathrm{C}$ for $10 \mathrm{~min}$, the samples were analyzed by SDS-PAGE followed by autoradiography.

\section{LC-MS/MS analysis}

Each sample was injected via the autosampler of an Ultimate 3000 chromatography system (Thermo Fisher Scientific, Waltham, MA, USA), and subsequently washed with solvent A (0.1\% formic acid in water) on an Acclaim PepMap100 $\left(\mathrm{C}_{18}, 5 \mu \mathrm{m}, 100 \AA\right.$ pore size, $300 \mu \mathrm{m}$ i.d. and $5 \mathrm{~mm}$ length; Thermo Fisher Scientific). The peptides were separated at a flow rate of $300 \mathrm{~nL} / \mathrm{min}$ on a nano HPLC capillary column $\left(\mathrm{C}_{18}, 3 \mu \mathrm{m}, 100 \AA\right.$ pore size, 75 $\mu \mathrm{m}$ i.d. and $120 \mathrm{~mm}$ length; Nikkyo Technos, Tokyo, Japan) with the following gradient: 1\% solvent B (0.1\% formic acid and acetonitrile) for $3 \mathrm{~min}$; $1-35 \%$ solvent B for $30 \mathrm{~min}$; 40$90 \%$ solvent B for $2 \mathrm{~min}$; and $90 \%$ solvent B for $10 \mathrm{~min}$. The eluted peptides were introduced into a Q-Exactive orbitrap mass spectrometer (Thermo Fisher Scientific) controlled by Xcalibur 4.2 software and measured in a scan range between $\mathrm{m} / \mathrm{z} 350$ to 1,500 with a resolution of 70,000 at $\mathrm{m} / \mathrm{z} 200$ for MS1. The 10 most abundant ions with charge state from $2^{+}$to $7^{+}$were subject to MS/MS with an automatic gain control target of $1.0 \mathrm{e}^{5}$, a maximum injection time of $60 \mathrm{~ms}$, and isolation windows of $\mathrm{m} / \mathrm{z}$ 1.6. The ions were fragmented via higher energy collision dissociation at normalized collision energy 27. MS2 scan had a resolution of 17,500 at $\mathrm{m} / \mathrm{z} 200$. 
MS and MS/MS spectral data were analyzed using the Proteome Discoverer 2.3 software (Thermo Fisher Scientific). In short, MS raw files were analyzed by Sequest HT. A mass tolerance $7 \mathrm{ppm}$ for precursor ions and 0.02 Da for product ions were set. The peak lists of precursor and product ions were assigned against to tryptic peptides of TpkD supplemented with cRAP database to avoid misassignment. A maximum of 2 miscleavage sites were allowed for trypsin digestion. 


\section{Supplementary Tables}

Table S1. The list of primers used for construction of the plasmid for the mutants.

\begin{tabular}{cl}
\hline Primer name & \multicolumn{1}{c}{ Sequences } \\
\hline W55F_fw & ccgggaccgggagctttttgtggggcgaaggctcg \\
W55F_rv & cgagccttcgccccacaaaaagctcceggtcccgg \\
W93F_fw & ggaggagctcggccgctttatggggaaaagccccg \\
W93F_rv & cggggcttttccccataaagcggccgagctcctcc \\
W191F_fw & cgggatcatcctctactttatgctcaccggggagc \\
W191F_rv & getcccoggtgagcataaagtagaggatgatcceg \\
\hline
\end{tabular}

Table S2. Thermodynamic parameters of the urea-induced unfolding of TpkD and the mutants. $^{\mathrm{a}}$

\begin{tabular}{|c|c|c|c|c|c|c|c|}
\hline \multirow{2}{*}{\multicolumn{5}{|c|}{ Three-state unfolding }} & \multirow{2}{*}{\multicolumn{3}{|c|}{ I --> U }} \\
\hline & & & & & & & \\
\hline & ATP & $\begin{array}{c}m_{\mathrm{I}-\mathrm{N}} \\
(\mathrm{kJ} / \mathrm{mol} / \mathrm{M})\end{array}$ & $\begin{array}{c}\Delta G_{\mathrm{I}-\mathrm{N}} \\
(\mathrm{kJ} / \mathrm{mol})\end{array}$ & $\begin{array}{c}C_{\mathrm{mI}-\mathrm{N}} \\
(\mathrm{M})\end{array}$ & $\begin{array}{c}m_{\mathrm{U}-\mathrm{I}} \\
(\mathrm{kJ} / \mathrm{mol} / \mathrm{M})\end{array}$ & $\begin{array}{c}\Delta G_{\mathrm{U}-\mathrm{I}} \\
(\mathrm{kJ} / \mathrm{mol})\end{array}$ & $\begin{array}{l}C_{\mathrm{mU}-\mathrm{I}} \\
(\mathrm{M})\end{array}$ \\
\hline Wild type & - & $4.9 \pm 0.6$ & $21.9 \pm 2.5$ & $4.5 \pm 0.7$ & $6.7 \pm 0.5$ & $45.5 \pm 3.3$ & $6.8 \pm 0.7$ \\
\hline W55F & - & $6.4 \pm 1.2$ & $27.2 \pm 4.8$ & $4.3 \pm 1.1$ & $5.8 \pm 0.4$ & $37.4 \pm 2.8$ & $6.4 \pm 0.7$ \\
\hline W93F & - & $5.5 \pm 0.4$ & $22.5 \pm 1.3$ & $4.1 \pm 0.4$ & $5.7 \pm 0.3$ & $37.5 \pm 2.2$ & $6.6 \pm 0.5$ \\
\hline \multicolumn{8}{|c|}{ Two-state unfolding } \\
\hline & & \multicolumn{3}{|c|}{$\mathrm{N}-->\mathrm{U}$} & & & \\
\hline & ATP & $\begin{array}{c}m_{\mathrm{U}-\mathrm{N}} \\
(\mathrm{kJ} / \mathrm{mol} / \mathrm{M})\end{array}$ & $\begin{array}{c}\Delta G_{\mathrm{U}-\mathrm{N}} \\
(\mathrm{kJ} / \mathrm{mol})\end{array}$ & $\begin{array}{c}C_{\mathrm{mU}-\mathrm{N}} \\
(\mathrm{M})\end{array}$ & & & \\
\hline Wild type & + & $13.7 \pm 3.4$ & $97.4 \pm 23.7$ & $7.1 \pm 2.5$ & & & \\
\hline W55F & + & $9.3 \pm 1.5$ & $64.1 \pm 10.1$ & $6.9 \pm 1.6$ & & & \\
\hline \multirow{2}{*}{ W191F } & - & $4.8 \pm 0.5$ & $31.7 \pm 2.8$ & $6.6 \pm 0.9$ & & & \\
\hline & + & $6.4 \pm 0.3$ & $42.2 \pm 2.1$ & $6.6 \pm 0.5$ & & & \\
\hline
\end{tabular}

a These parameters (with standard deviations) were obtained from the data of the fluorescence average wavelength. 


\section{Supplementary Figures}

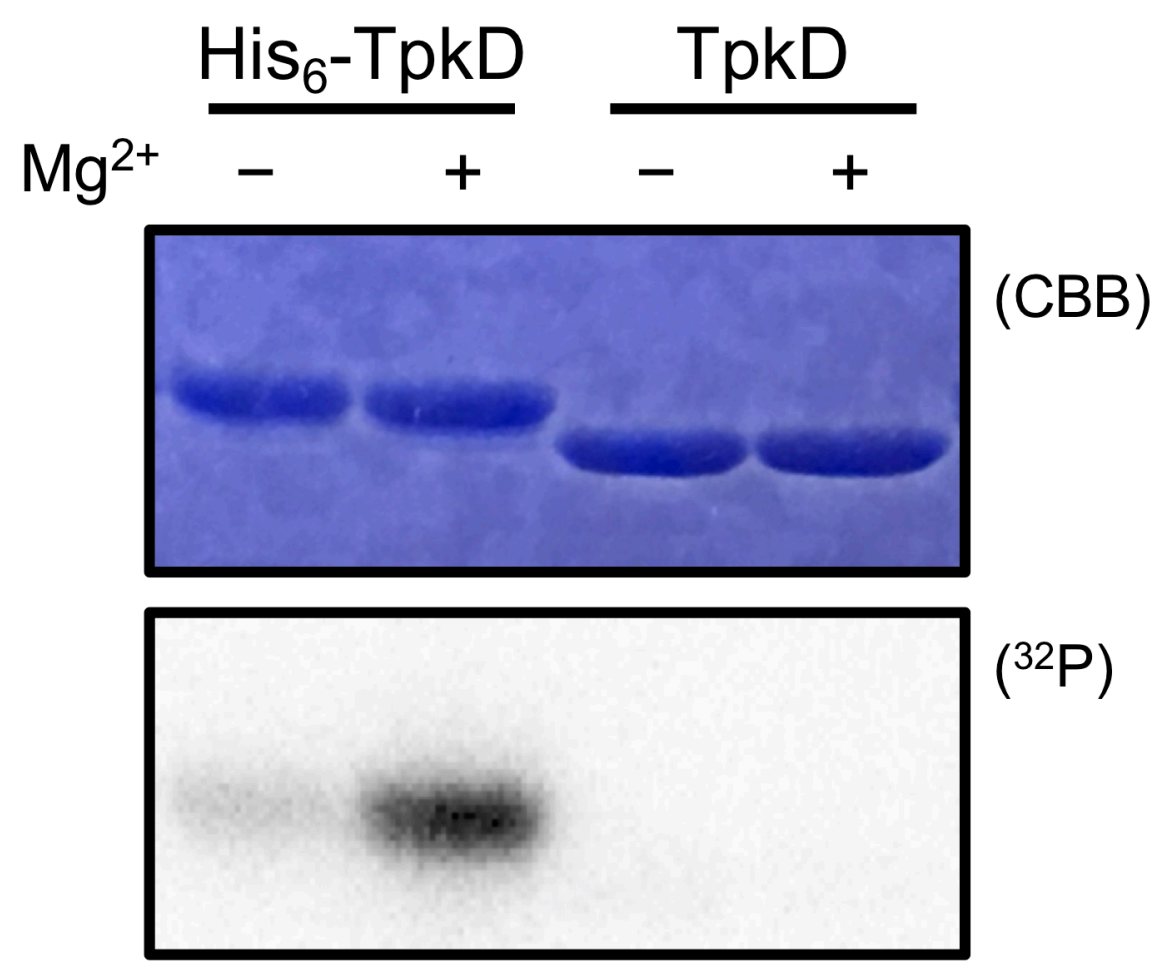

Fig. S1. Autophosphorylaion activity of $\mathrm{His}_{6}-\mathrm{TpkD}$ and $\mathrm{TpkD}$. The assay was performed in the presence or absence of $5 \mathrm{mM} \mathrm{MgCl}_{2}$. Other experimental conditions are described in the Supplementary Methods. The gel was stained with CBB (upper panel) and autoradiographed (lower panel).

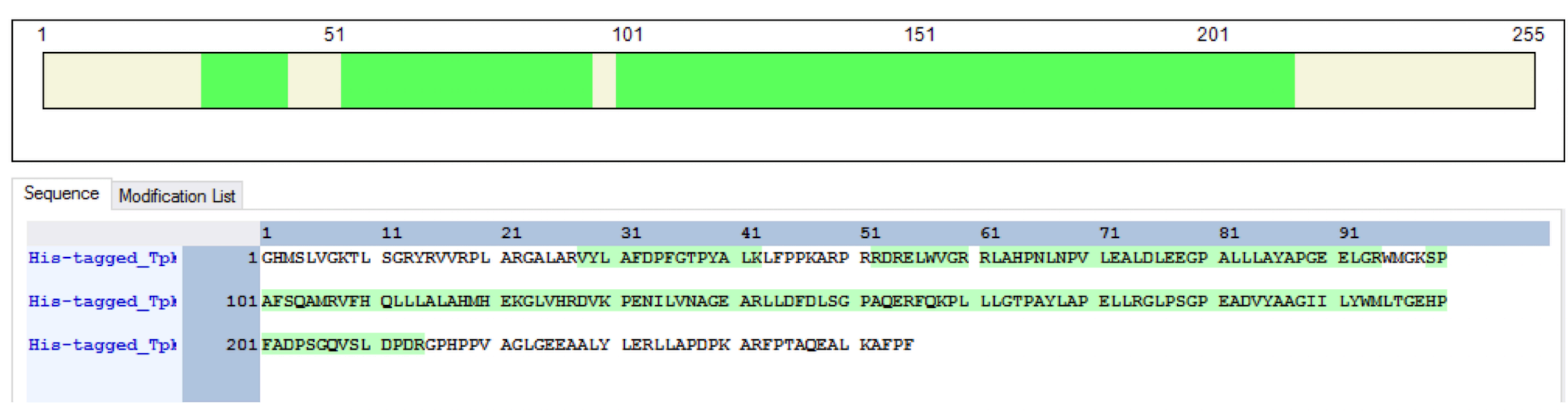

Fig. S2. Identification of the $20 \mathrm{kDa}$ fragment from trypsin-treated TpkD in LC-MS/MS analysis. The identified regions are colored green in the TpkD sequence. Note that the first two residues (Gly-His) are from the His-tag and the 3rd Met is the original N-terminal residue of TpkD. 


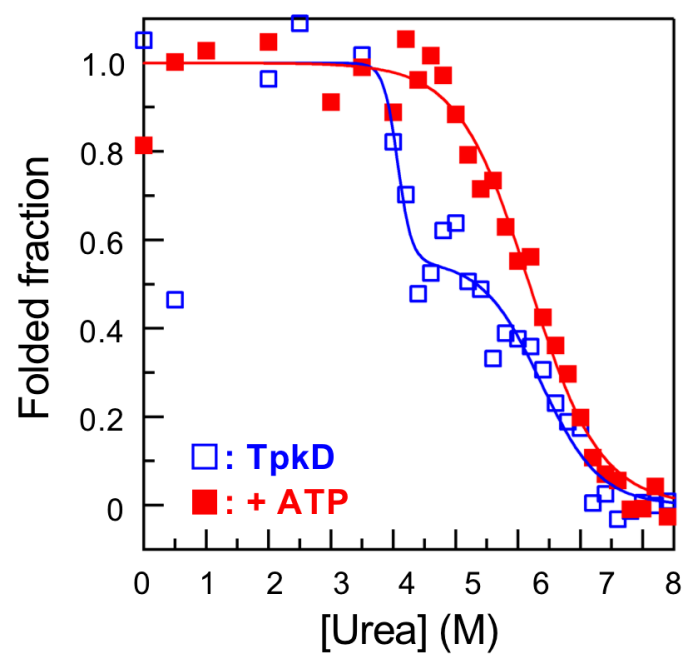

Fig. S3. Denaturation curves based on CD intensity at $222 \mathrm{~nm}\left([\theta]_{222}\right)$ with or without $1 \mathrm{mM}$ ATP. 